\title{
Search Engine Behavior and Satisfaction:A Survey-Based Study among Users in Malaysia
}

\author{
Annegret M. Gross \\ annegret.gross[at]ymail.com
}

\begin{abstract}
The birth of the internet in the 1990's did not only change the way of how people communicate but also of how they access information. The amount of information that the internet offers is enormous, it grows by the day and can be retrieved at any time - only technical issues may interrupt its constant availability. That is why the World Wide Web is nowadays the first port for information retrieval. Information retrieval tools are indispensible in this environment. They filter the information mass and allow users to locate the desired information.Search engines are one of the most popular web services.Most of what is known about user search engine behavior comes from query log analysis and clickthrough analysis. Only few studies have so far examined search engine behavior and user satisfaction from a mere user perspective.The number still shrinks when it comes to narrowing down the research focus on users of a specific country or language community. This study investigates user search engine behavior and satisfaction from the perspective of Chinese-speaking users in Malaysia. A survey made among 62 Malaysian Chinese users assesses search engine usage frequency, preferred search engines, and user priorities for search engine selection. It also assesses linguistic aspects such as preferred search language and search engine interface language. The study evaluates the users' satisfaction with the search results in general and the search results on the first page, with the search results of English and Chinese search queries, andthe necessity for query rephrasing. Where possible, the study compares the results with existing studies conducted in Malaysia.
\end{abstract}

Keywords: internet, information retrieval, search engine behavior, user satisfaction, Malaysia

\section{Introduction}

Comprehending and assessinguser interactions and satisfaction with search engines are important aspects of evaluating information retrieval on the internet. This work represents an investigative study of search engine behavior and satisfaction from the perspective of the users themselves. The target group considered in the test are Chinese-speaking users in Malaysia. The test aims at assessing the specific needs and habits of this language community when using an information retrieval system. Although recent years have shown anemerging approach of analyzing search engine log data or conducting controlled experiments to find out about user interactions, predict search success and searcher satisfaction, the number of user-perspective research of web search behavior and satisfaction that so far exists is still low. The motivation of the study is therefore to investigate how the users evaluate their own information search behavior and judge the quality of their search experience. The study is the first to provide results about graded user search satisfaction on a multi-level scale in different aspects of information retrieval. The analyzed aspects are the user satisfaction with the search results in general, the search results on the first page, and the search results obtained with English andChinese search queries. It is also the first study to examine graded search interactions, such as the necessity for query rephrasing, anduser preferencesas to search query language, search engine interface language, and priorities for search engine selection. The study results intend to make a contribution to the evaluation of user search behavior and qualitative search experience obtained by subjective user assessment.

\subsection{Population and Languages}

Malaysia has around 30 million inhabitants. The population is composed of three main ethnics groups: the Malays, the Chinese, and the Indians. After the Malays, the Malaysian Chinese form the second largest ethnic group. The majority of them migrated to then British Malaya from South China between the nineteenth and the mid-twentieth century to escape the harsh economic conditions in China. They brought along their native languages,Hokkien, Hakka, Cantonese, Teochew, Hokchew, and Hainanese. However, most of these Chinese languages are weakening due to the lack of intergenerational transmission. The languages spoken by Chinese immigrants in Malaysia shift increasingly to Mandarin and English (Sim, 2012).

\subsection{Internet Usageand Search Engine Market}

DOI: $10.9790 / 0837-2109102232$

www.iosrjournals.org

22 | Page 
Malaysia's current internet penetration is $67.5 \%$, i.e., more than 20 million out of 30 million people in total are using the web. Malaysia makes up around $1.3 \%$ of the total of internet users in Asia (Internet World Stats, 2016). Malaysia's search engine market is shared mainly between five search engines, as shown in Figure 1. The dominating search engine is Google, which holds a market share of more than 91\%. Far behind follow Yahoo with about 4\%, and Bing and Ask.com (formerly Ask Jeeves) with about 2\% each.Baidu is on last place with $0.12 \%$ (Stats Monkey, 2014). Baidu is the only Asian search engine in the listing. It was founded in 2000 by Chinese web services company Baidu, Inc. All other search engines are international.

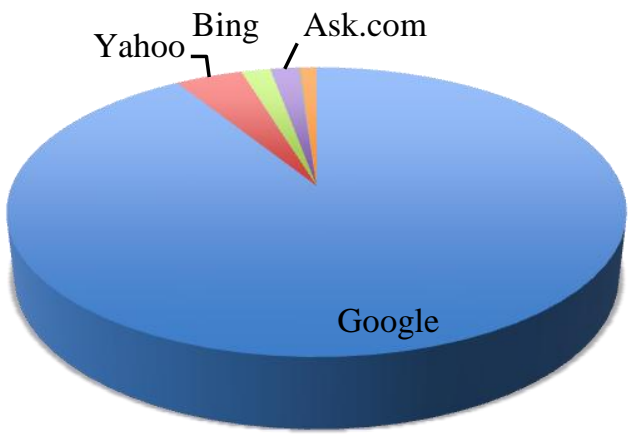

Google: $91.14 \%$

Yahoo: $4.17 \%$

Bing: $1.82 \%$

Ask.com: $1.75 \%$

Baidu: $0.12 \%$

Others: $1 \%$

Figure 1. Malaysia search engine market share as of 2014.

\section{Literature Review}

User-perspective studies on search engine behavior and search satisfaction in Malaysia are very few. The field has become a topic of interest only recently. In 2015, two studies have been published that examine search behavior and satisfaction of undergraduate students at different Malaysians educational institutions. Yamin, Ramayah and Ishak (2015) conducted a study on 131 randomly selected final year undergraduate students, 93 female and 38 male. The vast majority of them were below 25 years old (96.2\%). Information about the ethnic background of the participants was not provided. The research dealt with the relationship between user search satisfaction and user search knowledge. The investigations revealed that user knowledge influences the search behavior of the users, and that search behavior has an influence on search satisfaction. The study also involved other aspects of user behavior, such as internet usage frequency and search engine usage frequency. The present study will refer to comparable points in the later sections, where applicable.

Nadzir, Wahab and Othman (2015) carried out a survey among 39 final year students of information technology at Universiti Utara Malaysia (UUM). 37 of the students were female and 2 male. Themajority were Malay (29 or $74.4 \%$ ). The second largest ethnic group was Bumiputra (12.8\%), and the remaining participants were made upof Chinese, Indian, and international students. The survey aimed at identifying the students' information seeking behavior when looking up information for their research projects. The questionnaire contained, among others, questions about search engine usage, information seeking frequency, search query usage, determining and selecting relevant information. One of the results of the study is that most students clearly prefer the internet to other electronic or non-electronic sources. The authors contribute that to the variety of information available on the internet. Other findings of that study will be discussed later where a comparison can be drawn to the present research.

\section{Test Design}

The study aims at assessing search engine behavior and satisfaction from the subjective perspective of the users. Therefore, a questionnaire was handed out to 62 participants, who answered the questions anonymously. Firstly, the questionnaire collected demographic information, such as gender, age, and native language of the respondents. Secondly, the questionnaire contained nine questions relating to the users' search engine behavior and satisfaction. These questions could be answered either by multiple choice or by a Likert five-point scale (LFPS). One question allowed several free answers. In particular, the questions referred tosearch engine usage frequency, preferred search engine, preferred search language, and search engine interface language. Assessed were also the necessity for query paraphrasing as perceived by the users, the satisfaction with the search results in general and the search results on the first page,the satisfaction with search results in English versus search results in Chinese, and the users' criteria for search engineselection. The survey results were analyzed, calculated into percentage values, and illustrated in tables and figures.

\section{Results}




\subsection{Demographic Information of the Survey Respondents}

Demographically, the 62 survey respondents were composed as shown in Table 1: 60\% of the respondents were male, $40 \%$ were female. The age range of the participants was relatively widespread. The majority (59\%) were school students aged between 14 and 17 years. The second largest group was aged between 26 and 35 years (24\%). 9\% were older than 35 years, and $8 \%$ were between 18 and 25 years. $51 \%$ of the participants were native speakers of a Chinese language. $41 \%$ thereof indicated their native language to beMandarin, and $10 \%$ named another Chinese variety. 44\% described themselves as native speakers of English, and 5\% as bilingual in English and Mandarin.

Table 1. Demographic information of the survey respondents

\begin{tabular}{|c|c|c|c|c|}
\hline Gender & $\begin{array}{l}\text { male } \\
60 \%\end{array}$ & $\begin{array}{l}\text { female } \\
40 \%\end{array}$ & & \\
\hline Age & 14-17 & $18-25$ & 26-35 & $>35$ \\
\hline & $59 \%$ & $8 \%$ & $24 \%$ & $9 \%$ \\
\hline Native language & English & Mandarin & $\begin{array}{l}\text { Other Chinese } \\
\text { language }\end{array}$ & $\begin{array}{l}\text { English and } \\
\text { Mandarin }\end{array}$ \\
\hline & $44 \%$ & $41 \%$ & $10 \%$ & $5 \%$ \\
\hline
\end{tabular}

\subsection{Search Engine Usage Frequency}

The first survey question aimed at finding out how often the respondents make use of a search engine. The question was to be answered by a Likert five-point scale from 'very frequently' to 'never'. As shown in Table 2, almost half $(48 \%)$ of the users indicated that they used a search engine 'very frequently', and another $40 \%$ described their search engine usage as 'frequent'.12\% said they used a search engine 'sometimes'. None of the respondents classified their search engine usage frequency as 'seldom' or 'never'. The fact that altogether $88 \%$ of the users access a search engine on a very frequent and frequent basis confirms the important role that search engines play in locating information on the Web.

Table 2. Search engine usage frequency

\begin{tabular}{ccccc}
\hline very frequently & frequently & sometimes & seldom & never \\
\hline $48 \%$ & $40 \%$ & $12 \%$ & $0 \%$ & $0 \%$ \\
\hline
\end{tabular}

The two 2015 studies conducted in Malaysia offer comparable points. Their results match with the findings of this study: both studies found thatmore than half of the respective usersaccess an information retrievaltool on a daily basis. In more detail, Yamin, Ramayah and Ishak (2015) asked their participants to rate their search engine usage frequency by choosing between 'every day', 'every week', 'more than once per month', or 'once per month'. Slightly more than half of the respondents (54.4\%) used a search engine on a daily basis, and $43.5 \%$ on a weekly basis. Only $2.3 \%$ of the respondents made use of a search engine several times or once $(0.8 \%)$ in a month. Nadzir, Wahab and Othman (2015) examined in their study how often the students searched information for their research projects and proposed a similar frequency rating. The majority (61.5\%) rated their information seeking frequency with 'every day', and 30.8\% with 'once a week'. Only $7.7 \%$ or 3 of the respondents searched 'once a month' or 'rarely'.

\subsection{Mostly Used Search Engines}

The second survey question aimed at identifying the users preferred search engine or preferred search engines. The respondents could name up to three web domains (URL) of search engines they favor. The results are illustrated in Figure 2. 


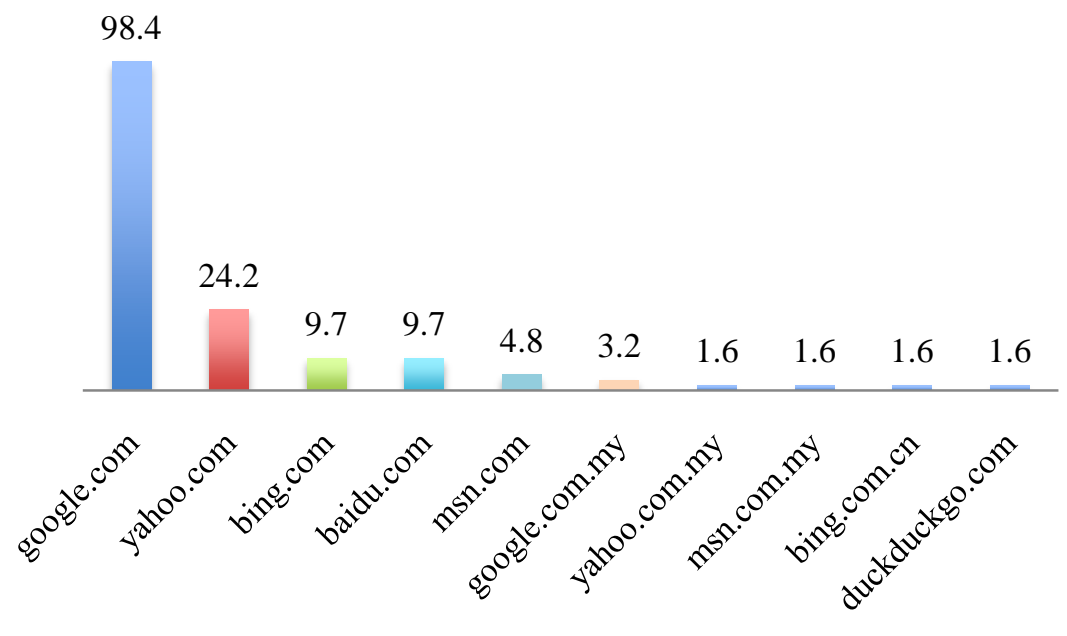

Figure 2. Mostly used search engines (multiple answers possible).

The vast majority of the respondents $(98.4 \%)$ named google.com as their search engine of choice. Thereof, $56.5 \%$ or 35 users, respectively,named exclusively google.com and no other search engine. Yahoo.com wasvoted second place with $24.2 \%$. Bing.com and baidu.com follow on third place with $9.7 \%$ each. Msn.com ranks fourth with $4.8 \%$. The Malaysian Google domain at google.com.my is on the fifth place with $3.2 \%$. The last place is shared bylocal domains of three international search engines -the Malaysian domains of Yahoo (yahoo.com.my) and of MSN (msn.com.my), the Chinese domain of Bing (bing.com.cn) - and the international search engine duckduckgo.com, with $1.6 \%$ each.

The fact that Google turned out to be the preferred search engine of the overwhelming majority of respondents is not surprising. After all, the search engine holds the largest market share in Malaysia and also worldwide. Interesting, however, is the distribution of the other search engines named by the survey respondents in comparison to the share those search engines hold on the Malaysian market. Yahoo, which has a market share of only $4 \%$ in Malaysia, obtained $24.2 \%$ of the user votes in the present survey. Bing and Baidu ranked third place in the survey with $9.7 \%$ each. On the Malaysian market, however, Bing holds a share of $1.82 \%$ only, and Baidu ranks even further behind with $0.12 \%$ market share. These three search engines are much more popular among the survey respondents than their market share would suggest. Furthermore, the search engine, which comes fourth place in terms of market share, Ask.com (1.75\%), was not listedamong the survey answers at all.

Figure 3compares the 2014 search engine market share statistics for Malaysia (Stats Monkey, 2014) with the mostly used search engines as named by the survey respondents. The illustration makes it obvious that Yahoo, Bing and Baidu hold greater percentages, by far, in the user preferences than in the official market.

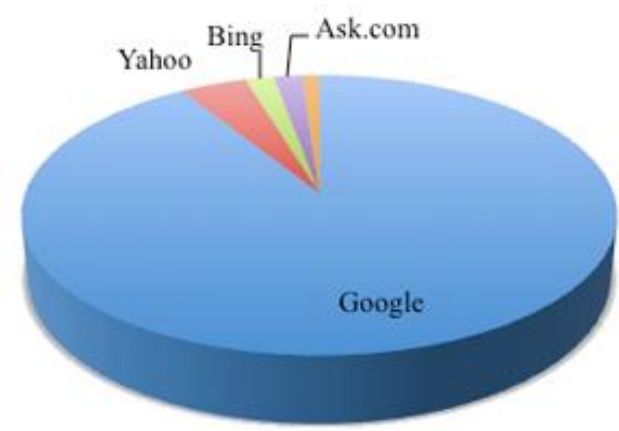

Malaysia search engine market share

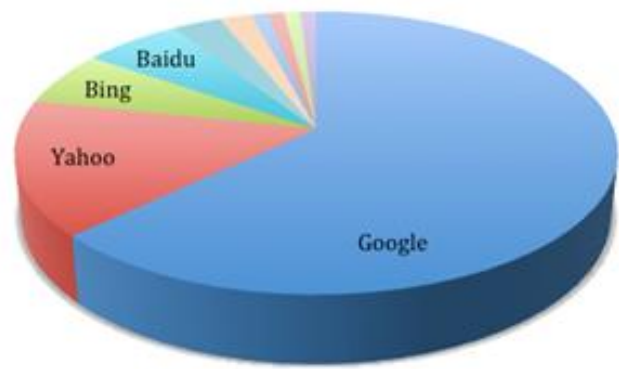

Preferred search engines in the survey

Figure 3. Graphical comparison of 2014 search engine market share in Malaysia and user search engine preferences as resulting from the present study.

User search engine preferences were investigated in only one recent study. The survey results obtained by Nadzir, Wahab and Othman (2015) are identical to those in the present study as to the fact that the majority 
of questioned students $(67 \%)$ also preferred to use Google as their main search engine. 19\% of the students opted for Google Scholar. Yahoo obtained $10 \%$ of the user votes. The remaining $4 \%$ were distributed among other search engines.

\title{
4.4 Priorities for Search Engine Selection
}

When investigating user search engine behavior from a user perspective and with Malaysia's search engine market statistics before the eyes, it is especially interesting to knowwhy users opt for a certain search engine. For this reason, the questionnaire asked the participants to name their priorities for search engine selection according to four criteria: precision of results, large number of retrieved documents, availability of search support tools, and fame and reputation of the search engine. The users were asked to rate these criteria according to their personal priorities from 1 ('very important') to 4 ('not so important'). Figure 4 shows the results.

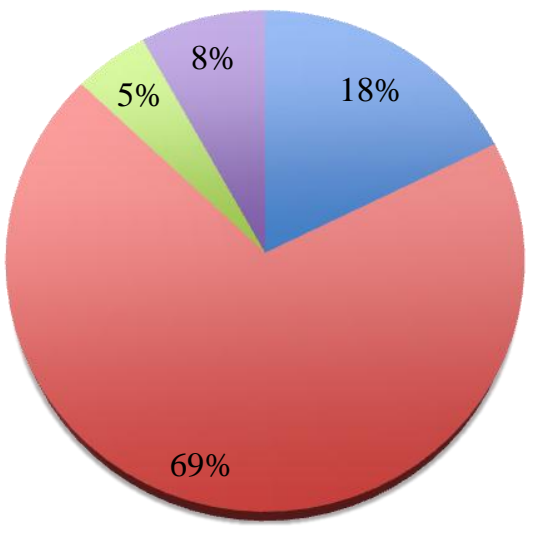

\author{
Large number of retrieved \\ documents \\ Precision of results \\ Fame and reputation of search \\ engines \\ Availability of search support \\ tools
}

Figure 4. User priorities for search engine selection.

Precise search results are the main factor toinfluence the users' decision for a certain search tool. Anoverwhelming $69 \%$ of the survey respondents consider the precision of results to be key. With about one fifth $(18 \%)$, the large number of retrieved documents stands on second place. Search support tools and fame and reputation of a search engine were considered less important by the respondents. The availability of search support received only $8 \%$ in the priority listing. Fame and reputation $(5 \%)$ do practically not play a role when deciding what search engine to use.

\subsection{Search Behavior}

Search behavior is defined as the micro level of behavior of a user interacting with an information retrieval system in order to seek information (Zhang, Anghelescu, Hermina,\& Yuan, 2005). A search process is composed of a sequence of actions. These include the problem identification, information need articulation, query formulation, and results evaluation (Sutcliffe \& Ennis, 1998). At the end stands the decision whether to repeat or to stop the search.Typically, users will stop searching when they have found the desired information and/or feel satisfied with the search results (Marchionini, 1995). As for search behavior, the present study was interested in how the users judge the necessity to use query paraphrasing in order to find the information they are looking for, and whatlanguage they mostly use for search queries and for their search engine interface.

\subsubsection{Necessity for Query Rephrasing}

After having identified the search task, the second step is to formulate the search query. The formulation of the search query is considered vital for the quality of the search results. The length of the search query also has an influence on the quality of the results. Beg and Ahmad (2007) distinguish between broad queries and narrow queries. Broad queries contain only a single or very few search terms and tend to be used by information seekers who are novice to a subject. Barsky and Bar-Ilan (2005) also note that users who are unfamiliar with a topic use short queries at the beginning of the search process. Narrow queries or long queries contain multiple qualifying terms and are entered by more versed users. A long query can substantially improve the quality of the information retrieved (Shapiro \&Taksa, 2003). If a search query does not bring about the desired information, usersmight reformulate the search term. This modification of a search query to satisfy the same information need is referred to as query rephrasing or reformulation (Shapiro \&Taksa, 2003; Huang \&Efthimiadis, 2009). 
Examples of query rephrasing are word reorder, removal or addition of words, word stemming, acronym formation and expansion, abbreviation, word substitution, spelling correction, modifications in punctuation and whitespaces, as well as semantic rephrasing. The combination of several of the before-mentioned reformulation strategies is referred to as multi-rephrasing. The most effective strategies seem to be the addition and removal of words, word substitution, acronym expansion, and spelling correction (Huang \&Efthimiadis, 2009).

In the present study, the participants were to indicate on a Likert five-point scale (LFPS) how frequently they faced the need to rephrase their search query in order to find the desired information on the web. Figure 5 illustrates the necessity for query rephrasing during a search process as perceived by the questioned users in Malaysia.

always $\square$ often $\square$ sometimes $\square$ seldom never

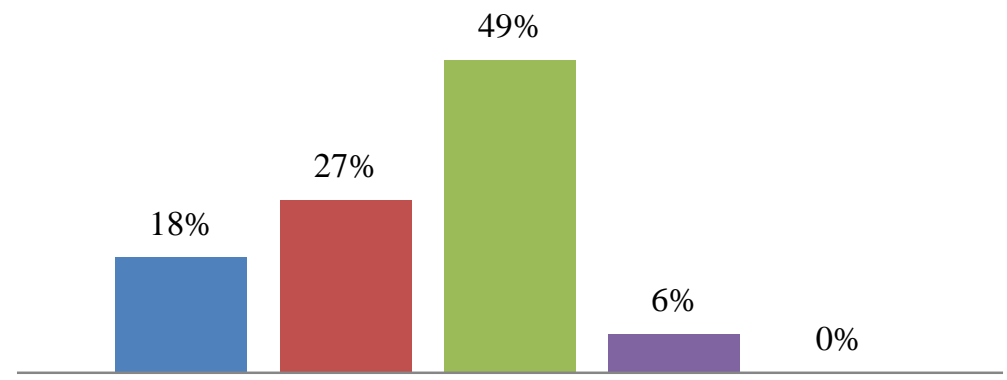

Necessity for query rephrasing

Figure 5. Necessity for query rephrasing as perceived by the users.

Figure 5 shows that the majority of answers gather in the middle area: approximately half (49\%) of the surveyed users see it 'sometimes' necessary for them to reformulate the search query. The second largest part (27\%) 'often'modifiesa search term, and 18\% 'always' use query rephrasing to fulfill their information need. Only $6 \%$ make 'seldom' use of query reformulations. None of the participantsselected 'never'. The results reveal that quite a large part of the respondents seem to be aware of the benefits of query reformulation strategies and make use of them on an averagebasis. Interestingly, all users who indicated to use rephrasing only 'seldom' are aged below eighteen. One possible explanation for this is that these users might notyet be so experienced with information retrieval methods and/or not so demanding as to specificity and quality of the information retrieved.

\subsubsection{Mostly Used Search Query Language and Search Engine Interface Language}

The present study also examined twolanguage-related aspects of search behavior. One question asked the survey participants what language they mostly use in order to seek information on the internet. Another question inquired the participants about their mostly used search engine interface language. All majorinternational search engines (Google, Yahoo, Bing, MSN etc.) allow the users to choose the language they want the search engine to be displayed in.Hence, the answer options for these two questions were 'English', 'Chinese', and 'it varies'.

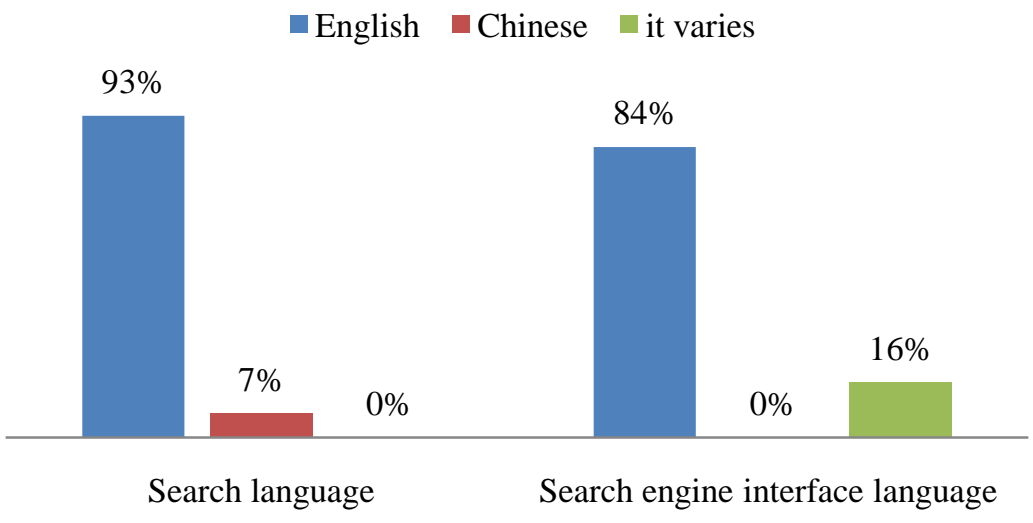

Figure 6. Mostly used search query language and search engine interface language. 
Figure 6illustrates clearly that the vast majority of respondents prefer to search in English language (93\%), and they also prefer to see their search engine displayedin English (84\%). Only very few of the surveyed users $(7 \%)$ tend to look up information in Chinese. None of the respondents use both languages variably for their information seeking activities. These results show that most of the altogether $51 \%$ survey participants, who are native speakers of Chinese, use the English language to satisfy their information seeking needs.

As for the mostly used search engine interface language, things look slightly different: none of the respondents use the search engine interface exclusively in Chinese. However, $16 \%$ of the users have their search engine sometimes displayed in English and sometimes in Chinese. Interestingly, nearly all respondents, who specified a varying interface language, were native speakers of Chinese. Only one person among them was a native speaker of English. This suggests that there is a relation between preferred search engine interface language and native language.

\subsection{User Satisfaction}

Search satisfaction is considered one of the indicatorsto determine whether userswere successful in fulfilling their information need (Zoe \&DiMartino, 2000). There aredifferent definitions of how search satisfaction is achieved. Tabatabai and Shore (2005) evaluate user satisfaction from a time perspective: if users find the desired information within a time frame of around 30 minutes, they are satisfied with the search results. Beg and Ahmad (2007), on the other hand,focus on the actions the user undertakes with the search results. They believe that user satisfaction can be gaugedby the sequence in which users examine the results, and whether or not they print, save, bookmark, e-mail, or copy-and-paste a portion of a relevant document. Topi and Lucas (2005) suggest that search performance in terms of accuracy and time, and the searcher's attitude and confidencehave an influence on the overall search satisfaction. Furthermore, as already mentioned above, query formulation also plays an important role when it comes to achieving search satisfaction. Knowledge of how to formulate and rephrase a query benefits the user (Huang and Efthimiadis, 2009). The present study investigated search satisfaction as perceived by the users in three aspects: firstly, the users' general satisfaction with the search results. Secondly, the users' contentment with the search results on the first page. Thirdly, the users' satisfaction with English search query results versus Chinese search query results.

\subsubsection{User Satisfaction with the Search Results}

The first question relating to user satisfaction aimed at finding out from the participants how satisfied they are with the search results in general. The subjectively perceived degree of satisfaction was to be indicated on a five-point scale from 'highly satisfied' to 'highly dissatisfied'. Figure 7 illustrates the survey results.

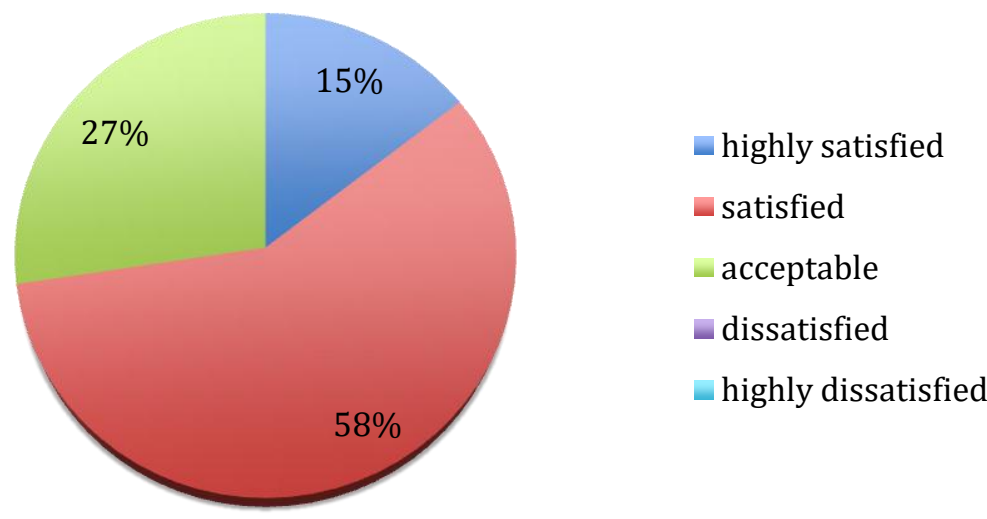

Figure 7. User satisfaction with search engine results in general.

The majority of the users (58\%) describe their search experience as satisfying. 15\% are 'highly satisfied' with the search results. About a quarter (27\%), however, consider their general search experience only 'acceptable'. None of the surveyed users finds the search results dissatisfactory or highly dissatisfactory. As to date, there is no comparable Malaysian study on user satisfaction with search engine results. Yamin, Ramayah and Ishak (2015) only note in their 2015 study that a higher knowledge of search query formulation leads to a higher search satisfaction. 


\subsubsection{User Satisfaction with the Search Results on the First Page}

The general opinion about the consultation of search engine results seems to be that users rarely view more than the results on the first page (Jansen, Spink, Bateman, \&Saracevic, 2000; Spink \& Jansen, 2004; Joachims, Granka, Pan, Hembrooke, \& Gay, 2005; van Deursen \& van Dijk, 2009; Schwartz, 2014).The quoted numbers vary. Spink and Jansen (2004), for instance, found that over 50\% of the users do not access results beyond the first page. According to van Deursen and van Dijk (2009), on the other hand, 91\% of the searchers do not look beyond the first results page, and over 50\% do not go past the first three results on the first page. Further studies found that search engine users tend to view only those results on the first page, which are visible without having to scroll down. A majority of users only views and clicks the top two results returned by a search engine(Granka, Joachims, \& Gay, 2004; Joachims et al., 2005). In a recent analysis of the clickthroughdata from Google, Schwartz (2014) comes to the conclusion that the results on the first page get around $71 \%$ of the clicks, results on page two and three only about $6 \%$. The first five links on the first page alone account for $68 \%$ of the clicks, and results from 6 to 10 for only $3.37 \%$.

The present study aimed atcollecting information on how the search engine users themselves perceive their satisfaction with the search results on the first page. The survey respondents were asked to indicate how often they are satisfied with the results on the first page and do not look any further on a five-point scale from 'always' to 'never' (Figure 8).

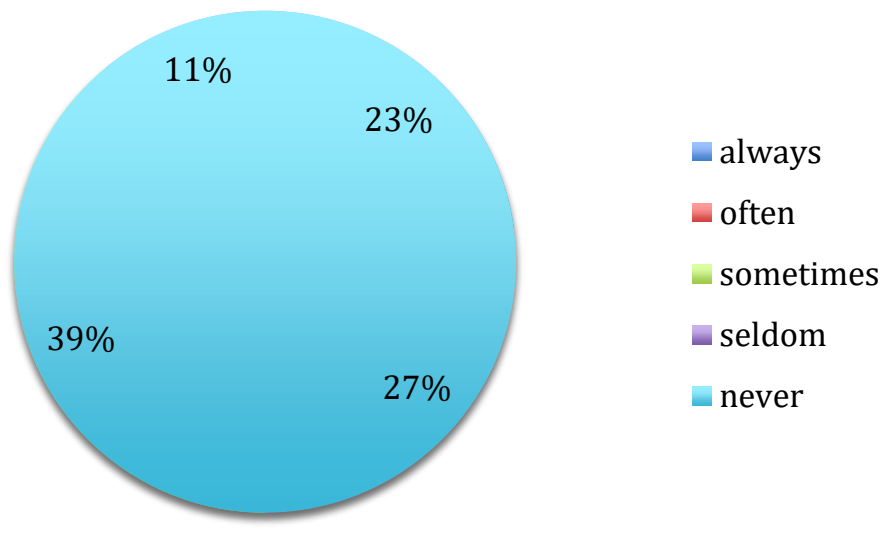

Figure 8. User satisfaction with search results on the first page.

The survey answersrevealthat the users do not categorically consider the search results on the first page to be satisfactory for them to stop the information seeking process. For the majority of respondents (39\%), the first page satisfies only 'sometimes' their information need. $27 \%$ of the users find the results on the first page 'often' sufficient. $11 \%$ say they are 'seldom' satisfied with the results onpage one. Only about a quarter (23\%) 'always'finds the desired information on the first page. Altogether, the survey results indicate that a high percentage of the users, according to their self-assessment, tend to consult more than only the first results page during their information seeking process.

\subsubsection{User Satisfaction with English Search Query Results versus Chinese Search Query Results}

The last survey question aimed at assessing the users' satisfaction with search results retrieved with English search query as compared to search queries in Chinese. To do so, the respondents were asked to rate on a fivepoint scale how often they think to obtain better search results with English search queries. The survey results are illustrated in Figure 9. 


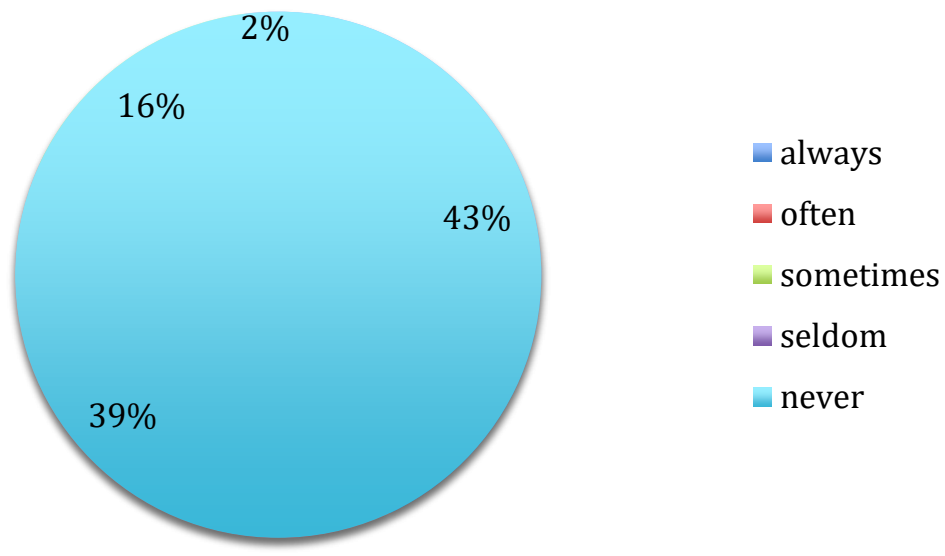

Figure 9. User satisfaction with English search query results versus Chinese search query results.

The survey results show that great parts of the users believe to obtain better results when using English search queries. $43 \%$ of the respondents perceive the quality of the results to be 'always' better when searching in English. An almost equally great part (39\%) believe that English search queries 'often' lead to better search results. $16 \%$ feel that English queries 'sometimes' satisfy their information need better than Chinese search queries. Only $2 \%$ consider the results achieved with English queries to be 'seldom' better than Chinese query results. A total of $51 \%$ of Chinese native speakers were involved in the survey (see Section 4.1). Interestingly, for 38\% out of these Chinese native speakers, English search queries 'always' produce better results, and for another $38 \%$ they 'often' do so. $22 \%$ of the Chinese native speakers think to 'sometimes' get better results with English queries, and only 2\% ticked 'seldom'. These results about user satisfaction with English search queries may explain why 93\% of the respondents prefer to search in English, and only 7\% tend to search in Chinese (see Section 4.5.2).

\section{Conclusion}

This study focused on search engine behavior and satisfaction from a user perspective of Chinese-speaking users in Malaysia. First of all, the study results confirm the importance of search engines as information retrieval tools on the web: $88 \%$ of the respondentsuse a search engine on a frequent or a very frequent basis. Google.com turned out to be the most popular portal for information seeking. More than $98 \%$ of the users named it amongtheir preferred search engines or even as their only search engine used.The international search engines Yahoo, Bing, and Baidufollowed after Google, but with by far less popularity percentage-wise. The users seem to be aware of query reformulation strategies and their benefits. Alarge part makes use of them on a regular $(45 \%)$ or occasional (49\%) basis. The users clearly favor English search queries (93\%) to search queries in Chinese, because they believe to obtain better results with English search queries. This also concerns Chinese native speakers. In this respect, the results reveal that there is no relation between native language and preferred search query language. On the other hand, the results suggest a relation between preferred search engine interface language and native language. While the majority of users (84\%) prefer to have their search engine displayed in English, nearly all respondents (16\%), who use their search engine interface alternately in English and Chinese, are native speakers of Chinese. 60\% of the participants described their general search experience as satisfactory, $15 \%$ as highly satisfactory. Against the general opinion, most users do not stop browsing the search results after having viewed the first page or the first three to five links "above the fold."A great percentageof the users doesnot consider the search results on page one satisfactory to their information need and tends to go past the first results page during an information seeking session.

\section{REFERENCES}

[1] Barsky, E. \& Bar-Ilan, J. (2005).From the search problem through query formulation to results on the web.Online Information Review, 29(1), 75.

[2] Beg, M.M.S. \& Ahmad, N. (2007).Web search enhancement by mining user actions.Journal of Information Science, 177(23), 5203-5218.

[3] Granka, L., Joachims, T., \& Gay, G. (2004). Eye-tracking analysis of user behavior in WWW 
search.Proceedings of the 27th Annual International ACM SIGIR Conference on Research and Development in Information Retrieval (SIGIR'04), 478-479.

[4] Huang, J. \&Efthimiadis, E.N. (2009). Analyzing and evaluating query reformulation strategies in web search logs. Proceedings of the 18thACM Conference on Information and Knowledge Management(CIKM'09), 77-86.

[5] Internet World Stats (2016). Asia internet use, population data and Facebook statistics. Retrieved from http://www.internetworldstats.com/stats3.htm

[6] Jansen, B.J., Spink, A., Bateman, J., \&Saracevic. T. (2000). Real life, real users, and real needs: A study and analysis of user queries on the Web. Information Processing and Management, 36(2), 207- 227.

[7] Joachims, T., Granka, L., Pan, B., Hembrooke, H. \& Gay, G. (2005). Accurately interpreting clickthrough data as implicit feedback. Proceedings of the 28th Annual International ACM SIGIR Conference on Research and Development in Information Retrieval (SIGIR'05), 154-161.

[8] Marchionini, G. (1995). Information seeking in electronic environments. Cambridge, MA: Cambridge University Press.

[9] Nadzir, M.M., Wahab, A.A. \& Othman, N. (2015). Undergraduates' needs and seeking behaviour: a preliminary study. Journal of Education and Vocational Research, 6(2), 48-54.

[10] Schwartz, B. (2014). A new click through rate study for Google organic results. Retrieved from http://marketingland.com/new-click-rate-study-google-organic-results-102149

[11] Shapiro, J. \&Taksa, I. (2003). Constructing Web search queries from the user's information need expressed in a natural language. Proceedings of the 2003 ACM Symposium on Applied Computing (SAC'03), 1157-1162

[12] Sim, T.W. (2012). Why are the native languages of the Chinese Malaysians in decline?.Journal of Taiwanese Vernacular 4(1), 62-95.

[13] Spink, A. \& Jansen, B. J. (2004). A study of Web search trends. Webology, 1(2). Retrieved from http://www.webology.org/2004/v1n2/a4.html

[14] Stats Monkey (2014). Malaysia Search Engine Market Share, Usage Statistics - 2014. Retrieved from https://www.statsmonkey.com/sunburst/18436-malaysia-search-engine-market-share-usage-statistics2014.php

[15] Sutcliffe, A. \& Ennis, M. (1998).Towards a cognitive theory of information retrieval.Interacting with Computers, 10(3), 321-351.

[16] Tabatabai, D. \& Shore, B.M. (2005). How experts and novices search the web. Library and Information Science Research, 27(2), 222-248.

[17] Topi, H., \& Lucas, W. (2005). Searching the Web: operator assistance required. Information Processing \& Management, 41(2), 383-403.

[18] van Deursen, A.J.A.M., \& van Dijk, J.A.G.M. (2009). Using the internet: skill related problems in users' online behavior. Interacting with Computers. doi:10.1016/j.intcom.2009.06.005

[19] Yamin, F.M., Ramayah, T. \&Ishak, W.H.W. (2015). Does user search behaviour mediate user knowledge and search satisfaction?.International Journal of Economics and Financial Issues, 5(Special Issue), 3439.

[20] Zhang, X., Anghelescu, H., Hermina, G.B. \& Yuan, X. (2005). Domain knowledge, search behaviour, and search effectiveness of engineering and science students: an exploratory study. Information Research, 10(2), 1-17.

[21] Zoe, L.R., \&DiMartino, D. (2000). Cultural diversity and end user searching: an analysis by gender and language background. Research Strategies, 17(4), 291-305.

\section{List of Tables}

Table 1 .

Table 2.

\section{List of Figures}

Figure 1.

Figure 2.
Demographic information of the survey respondents.

Search engine usage frequency.

Malaysia search engine market share as of 2014.

Mostly used search engines (multiple answers possible). 
Figure 3. Graphical comparison of 2014 search engine market share in Malaysia and user search engine preferences as resulting from the present study. [Left graph], based upon data from Stats Monkey (2014). Malaysia Search Engine Market Share, Usage Statistics - 2014. Retrieved fromhttps://www.statsmonkey.com/sunburst/18436-malaysia-search-engine-market-shareusage-statistics-2014.php

Figure 4. User priorities for search engine selection.

Figure 5. Necessity for query rephrasing as perceived by the users.

Figure 6. Mostly used search language and search engine interface language.

Figure 7. User satisfaction with search engine results in general.

Figure 8. User satisfaction with search results on the first page.

Figure 9. User satisfaction with English search query results versus Mandarin search query results. 